\title{
Lithium isotopic composition of biological reference materials and automated Li separation using PrepFAST MC $^{\text {TM }}$
}

\author{
LUCAS WEPPE ${ }^{1}$, FANNY THIBON ${ }^{1}$, MARYLINE \\ MONTANES $^{1}$, PHILIPPE TELOUK ${ }^{2}$, AURELIEN \\ VISCARDI $^{3}$ AND NATHALIE VIGIER ${ }^{4}$ \\ ${ }^{1}$ LOV- CNRS-Sorbonne Université \\ ${ }^{2}$ ENS Lyon \\ ${ }^{3}$ ESI FRANCE \\ ${ }^{4}$ LOV - CNRS - Sorbonne Université
}

Presenting Author: lucas.weppe@obs-vlfr.fr

Although Li isotopes are increasingly used to study biological systems [1-2] very few reference materials have been characterized yet, focusing mostly on carbonate phases [3]. The present study reports $\mathrm{Li}$ concentrations and $\mathrm{Li}$ isotopic compositions for six biological reference materials: TORT-2, TORT-3 (lobster hepatopancreas), DORM-2, DORM-4 (dogfish muscle), ERM-CE278k (mussel tissue), and SRM-1400 (bone ash). An in-house standard of Mediterranean plankton (PLKVLFR) was analyzed as well. The method used involve many replicate analyses, and a standard addition technique, allowing us to quantify precisely the various sources of uncertainties. Li concentrations range from $0.05 \mu \mathrm{g} / \mathrm{g} \mathrm{dw}$ (DORM-2) to $10.5 \mu \mathrm{g} / \mathrm{g}$ $\mathrm{dw}$ (PLK-VLFR). Sample purification for Li isotope analysis was made using AG-50-X12 resin columns. Li isotopic ratio was measured using the MC-ICP-MS Neptune Plus. The average $\delta^{7} \mathrm{Li}$ values are $30.98 \pm 0.47 \%$ for PLK-VLFR, $19.28 \pm 4.90 \%$ for DORM-2, $29.17 \pm 0.24 \%$ for DORM-4, $22.64 \pm 2.81 \%$ for TORT-2, $24.17 \pm 0.32 \%$ for TORT-3, $14.69 \pm 0.35 \%$ for ERM-CE278k, and $-1.72 \pm 0.48 \%$ for SRM-1400.

To optimize and speed up the routine chemical preparation for Li purification, we are developing a new automated method using PrepFAST MC ${ }^{\mathrm{TM}}$. The purification protocol is optimized by adapting the resin column size, eluents type, volume and molarity, and the solutions flow rates. First results are encouraging and in the near future, this new method will help to decrease the sample preparation time, leading to rapid isotope analyses of low-Li organic-rich samples, which is a necessary step for biological studies, requiring a significant number of replicates.

[1] Dellinger et al. (2018), GCA 236, 315-335 [2] Balter \& Vigier (2014), Metallomics 6, 582-586 [3] Bastian et al. (2018), GGR 42, 403-415. 